\title{
Robust Color Demosaicking With Adaptation to Varying Spectral Correlations
}

\author{
Fan Zhang, Xiaolin Wu, Senior Member, IEEE, Xiaokang Yang, Senior Member, IEEE, \\ Wenjun Zhang, Member, IEEE, and Lei Zhang, Member, IEEE
}

\begin{abstract}
Almost all existing color demosaicking algorithms for digital cameras are designed on the assumption of high correlation between red, green, blue (or some other primary color) bands. They exploit spectral correlations between the primary color bands to interpolate the missing color samples, but in areas of no or weak spectral correlations, these algorithms are prone to large interpolation errors. Such demosaicking errors are visually objectionable because they tend to correlate with object boundaries and edges. This paper proposes a remedy to the above problem that has long been overlooked in the literature. The main contribution of this work is a hybrid demosaicking approach that supplements an existing color demosaicking algorithm by combining its results with those of adaptive intraband interpolation. This is formulated as an optimal data fusion problem, and two solutions are proposed: one is based on linear minimum mean-square estimation and the other based on support vector regression. Experimental results demonstrate that the new hybrid approach is more robust and eliminates the worst type of color artifacts of existing color demosaicking methods.
\end{abstract}

Index Terms-Autoregressive model, color demosaicking, color saturation, digital cameras, linear minimum mean-square estimation (LMMSE), support vector regression (SVR).

\section{INTRODUCTION}

$\mathbf{I}$ $\mathrm{N}$ quest of low cost, compact size, and long battery life, most digital cameras use a single sensor array to capture color images. At each pixel position only one instead of three or more primary colors (e.g., red, green, and blue) is captured with a color filter array (CFA). The most commonly used CFA is that of Bayer pattern [1] that consists of a quincunx lattice of green samples interleaved with one square lattice of red samples and another square lattice of blue samples, as depicted by Fig. 1. The full color image is reconstructed by interpolating the missing color samples, a process commonly known as color

Manuscript received May 22, 2009; revised July 06, 2009. First published August 11, 2009; current version published November 13, 2009. This work was supported in part by the 111 Project of China, in part by a Natural Sciences and Engineering Research Council of Canada industrial research chair grant, and in part by the Hong Kong RGC General Research Fund (PolyU 5330/07E). The associate editor coordinating the review of this manuscript and approving it for publication was Dr. Gaurav Sharma.

F. Zhang, X. Yang, and W. Zhang are with the Institute of Image Communication and Information Processing, Shanghai Jiao Tong University, China (e-mail: fanzhang1985@gmail.com; xkyang@sjtu.edu.cn; zhangwenjun@sjtu.edu.cn).

$\mathrm{X}$. Wu is with the Department of Electrical and Computer Engineering, McMaster University, Hamilton, ON L8S 4K1 Canada (e-mail: xwu@ece.mcmaster.ca).

L. Zhang is with the Department of Computing, The Hong Kong Polytechnic University, China (e-mail: cslzhang@comp.polyu.edu.hk).

Color versions of one or more of the figures in this paper are available online at http://ieeexplore.ieee.org.

Digital Object Identifier 10.1109/TIP.2009.2029987

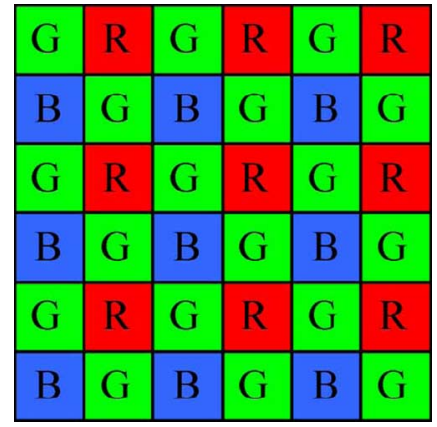

Fig. 1. Bayer pattern of color mosaic for digital cameras.

demosaicking, which is crucial to the visual quality of digital cameras.

A basic premise of CFA mosaic undersampling schemes for color reproduction is the following. Most scenes in nature comprise of pastoral colors, and highly saturated colors are rare, i.e., strong correlations exist between different spectral bands. Indeed, most existing color demosaicking algorithms, particularly those of competitive performance [2], [3], assume and exploit the spectral correlations when interpolating the missing color samples. In general, those demosaicking techniques that process different color bands in isolation are inferior to the interband approach. For a recent survey of, including a taxonomy, of existing demosaicking techniques please refer to [4].

Among previously published color demosaicking techniques, the primary-consistent soft-decision demosaicking (PCSD) [5] performs the best over a diverse set of color images (see Section $\mathrm{V}$ for comparison). The PCSD algorithm exemplifies an explicit and thorough use of spectral correlations in demosaicking. It assumes the difference signals between green and red and between green and blue to be low-pass, and estimates the color difference signals. The estimates of missing red and blue samples are anchored on the green band that has the least chance of aliasing in Bayer CFA. Moreover, PCSD imposes the same interpolation direction in all three color bands.

However, this work demonstrates that aggressive uses of spectral correlations in color demosaicking can sometimes backfire. When the assumption of high spectral correlations does not hold, for instances, in areas of highly saturated colors, and if large sensor noises are present, demosaicking methods overemphasizing spectral correlations can produce highly visible, objectionable color artifacts. Indeed, in such cases, the PCSD algorithm and other sophisticated ones [2], [3] become inferior to the classic method of Hamilton and Adams [6], while the latter could in turn be even worse than simple but more conservative bicubic interpolation. The isotropic separable 


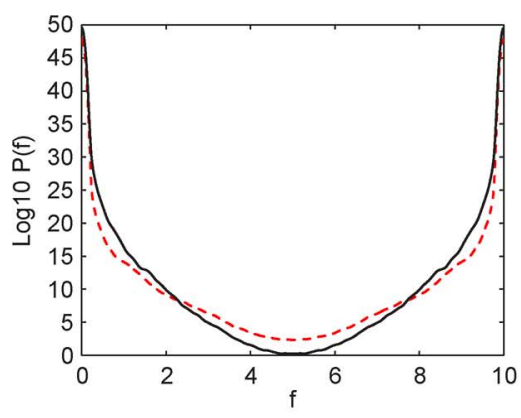

(a)

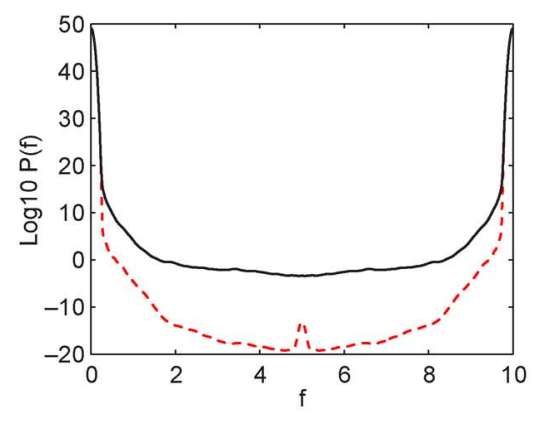

(b)

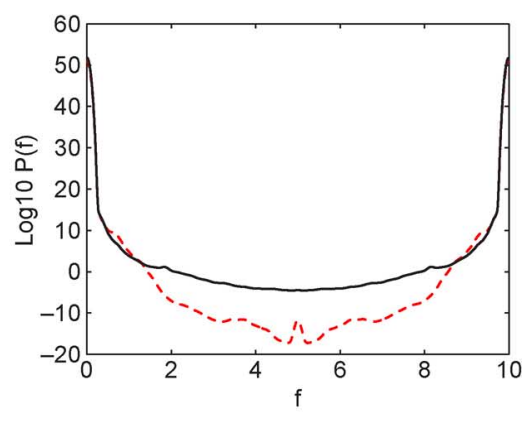

(c)

Fig. 2. Y, U, V power spectrums of Lena (solid line) and the girl-with-red-hat image (dotted line) in the Kodak set. (a) Y; (b) U; (c) V.

TABLE I

INTERBAND CORRELATIONS OF TwO IMAGE SETS

\begin{tabular}{|c|c|c|c|c|c|}
\hline \multirow{2}{*}{ Image set } & \multirow{2}{*}{ Statistic term } & \multicolumn{4}{|c|}{ Interband correlation coefficients } \\
\cline { 3 - 6 } & Mean & 0.872 & 0.906 & 0.767 & $\mathbf{0 . 8 4 8}$ \\
\hline \multirow{3}{*}{ Kodak } & Std. deviation & 0.131 & 0.115 & 0.191 & $\mathbf{0 . 1 4 6}$ \\
\hline \multirow{3}{*}{ Normal } & Mean & 0.675 & 0.720 & 0.558 & $\mathbf{0 . 6 5 1}$ \\
\cline { 2 - 6 } & Std. deviation & 0.321 & 0.343 & 0.284 & $\mathbf{0 . 3 1 6}$ \\
\hline
\end{tabular}

bicubic interpolator does not bet on a gradient direction by assuming spectral correlation as in [6] and [5] and, hence, avoid worst-case errors when the assumption is invalid.

The problem of overusing spectral correlation in color demosaicking was overlooked by many researchers in academia, ourselves included. The reason seemed to be that they were misled by a peculiarity of the popular Kodak set of test images that were commonly used to simulate CFA data and benchmark demosaicking performance. The spectral correlations of these test images are considerably higher (much smoother hue) than typical color images, probably due to some postprocessing. Let us compare the correlation coefficients $\rho_{G R}$ (between the green and red bands), $\rho_{G B}$ (between the green and blue), and $\rho_{R B}$ (between the red and blue) for the Kodak set and another set of 30 images randomly chosen from the JPEG, MPEG, SMPTE test sets and various internet sites. The averages of $\rho_{G R}, \rho_{G B}$, and $\rho_{R B}$ for the two test sets are tabulated in Table I. The Kodak set has substantially higher spectral correlations than normal. The table also reveals that the Kodak set has a significantly smaller standard deviation than normal ( 0.146 versus 0.316$)$ in spectral correlation. In other words, the Kodak set seems to include a quite narrow range of color images.

In a closer examination, we find that the power spectrums of the Kodak set in the $\mathrm{U}$ and $\mathrm{V}$ components have a much faster decay (much lower energy in high frequency) than normal. But strangely, this fast decay is not monotone, and the $\mathrm{U}$ and $\mathrm{V}$ power spectrums of each image in the Kodak set, without exception, have a spike at the highest frequency of the sampling rate, suggesting that these images are edge enhanced. This peculiarity is evident by comparing the power spectrums of two similar portrait images: Lena from the JPEG set and the girl-withred-hat image from the Kodak set, which are plotted in Fig. 2. All the above atypical characteristics of the Kodak set make it ill suited for simulation of CFA data. As a result, the reported performances of many previous papers are cast in doubt. Indeed, we find that on the aforementioned set of 30 typical color

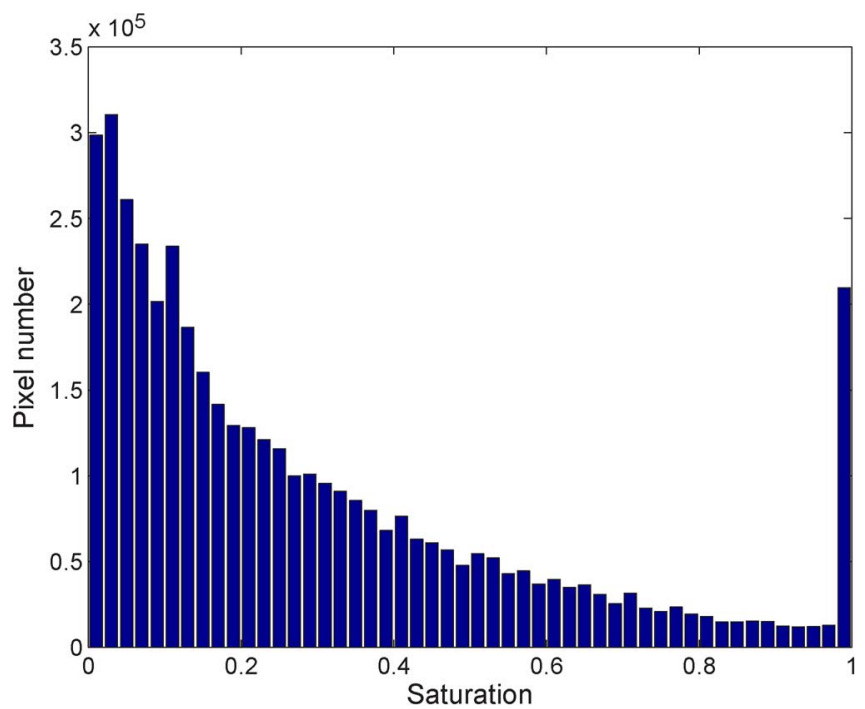

Fig. 3. Color distribution in saturation for Flickr images.

images, some of recent methods actually perform worse than the classic method of Hamilton and Adams [6]. More detailed results will be reported in Section V. To be fair, to Eastman Kodak Company, the so-called Kodak set was made publicly available not for color demosaicking research. The image set became a de-facto benchmark for evaluating demosaicking algorithms largely because academic researchers were not thorough enough to choose a good representative set of color images in their evaluations.

The current color demosaicking algorithms were geared for colors of low saturation. But this practice should be questioned. Although having a small probability in terms of pixel count, saturated colors are by no means pathological and they do appear in daily scenes, such as traffic and commercial signs (often in pure red, blue, or green), painted object surfaces, and vivid flowers. In Fig. 3, we plot the color distribution in terms of saturation for a random draw of 42 images from the popular internet image site Flickr. Note the peak of the distribution near the full saturation. Semantically speaking, saturated colors are typically the centers of attentions in an image composition. Demosaicking errors that correlate with the edges of saturated colors easily stand out and become annoying, and they can degrade the subjective image quality by a degree that is disproportional to error population. One of the authors observed the failures of existing color demosaicking methods to reproduce saturated colors, and 


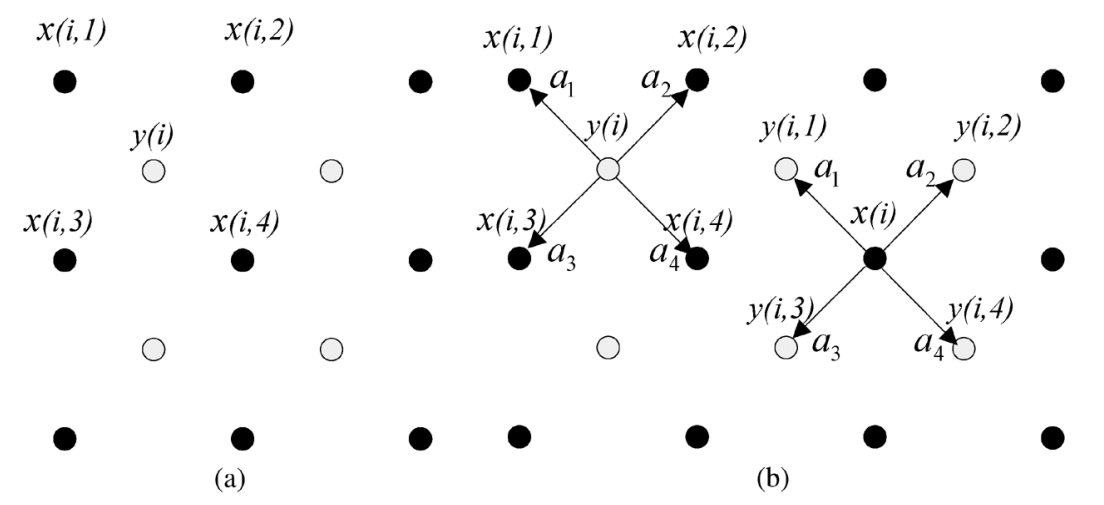

Fig. 4. (a) Spatial configuration in first pass of PARM. (b) PARM model parameters $\boldsymbol{a}=\left(a_{1}, a_{2}, a_{3}, a_{4}\right)$ in relationship to spatial correlations of pixels.

proposed a remedy of temporal demosaicking [7]. But this approach is only applicable to digital video cameras. Chang and Tan also observed that some demosaicking methods tended to produce artifacts in areas of weak spectral correlation [8], [9], and proposed a technique called EUSSC (effective use of spatial and spectral correlations). This is essentially an approach of demosaicking a CFA image into two estimated color images, under the assumptions of strong and weak spectral correlation, respectively. When assuming weak spectral correlation, EUSSC lessens the constraint on the smoothness of the band difference signal. At each spatial location, the local spectral correlation is estimated. This estimated correlation is then thresholded to determine which of the two demosaicked results to use.

The goal of this research is to lend a degree of universality to the existing color demosaicking technology for still cameras. Our main contribution is a new mechanism of adapting to varying spectral correlations in demosaicking. The idea is to judiciously combine spatial-spectral demosaicking with an adaptive intraband interpolation process. Pure spatial demosaicking is carried out separately in each of the color bands to produce supplementary estimates of missing samples. These intraband estimates are fused with the interband estimates produced by an existing demosaicking method to mitigate the bad artifacts of the latter. Spatial demosaicking is a problem of image interpolation in quincunx or square sublattice of the original image, depending on whether the green, or blue/red band is in question. The proposed adaptation mechanism is general, and it can work with any image interpolation algorithm. But the performance of the chosen interpolation algorithm affects the quality of spatial demosaicking and in turn the final fused result. For this reason, we choose the recent technique of soft-decision adaptive interpolation for its superior performance [10]. The key to the success of the proposed hybrid approach is how well the underlying data fusion problem can be solved. We propose two solutions of different complexity-performance tradeoffs: one is based on classic linear minimum mean-square estimation (LMMSE); the other computes the optimal fusion weight by support vector regression (SVR). The SVR technique, which is extensively studied in the machine learning literature, can incorporate preknowledge of a training set in the weighting of inter and intraband estimates. Therefore, the latter is more powerful and robust than the former. Simulation results verify the superior performance of the proposed techniques to existing methods, in both PSNR measure and perceptual image quality. Certain types of objectionable artifacts associated with existing color demosaicking methods are eliminated.

This paper is structured as follows. In Section II, we introduce the notions of intraband demosaicking via piecewise autoregressive modeling. Section III presents linear minimum mean-square fusion of intra and interband estimates of missing color samples. Section IV describes how these two different estimates can be combined by support vector regression to obtain a more robust estimate when the conditions of LMMSE do not hold. Section V gives the experimental results, and Section VI concludes.

\section{INTRABAND DEMOSAICKING VIA PIECEWISE AUTOREGRESSION}

When there is no spectral correlation to exploit, the better strategy is to perform spatial interpolation rather than spectral demosaicking based on erroneous assumption. The intraband demosaicking of three down-sampled bands in the Bayer CFA is a problem of image interpolation in quincunx or square sublattice of the original image, depending on whether the green, or blue/red band is in question. We solve this problem by a new image interpolation method based on piecewise autoregressive modeling (PARM) [10]. In this section, we sketch the main idea of the PARM technique and how it can be applied to intraband demosaicking.

First, consider the interpolation of the blue/red band that constitutes a square sublattice of the original image in the Bayer CFA. Let $I_{h}$ be the blue/red image to be estimated, and $I_{l}$ be the down-sampled blue/red image. Let $x(i) \in I_{l}$ and $y(i) \in I_{h}$ be the pixels of $I_{l}$ and $I_{h}$ respectively.

Interpolation of the missing pixels is carried out in two passes. In the first pass, those missing pixels $y(i) \in I_{h}$, whose four 8-connected neighbors are known observed pixels $x(i, t) \in I_{l}$, $t \in\{1,2,3,4\}$, are interpolated. This configuration is depicted in Fig. 4(a). The interpolation problem is posed as one of nonlinear optimization

$$
\begin{aligned}
\min _{\boldsymbol{a}, \mathbf{y}}\left\{\sum_{i \in W}\left\|y(i)-\sum_{t=1}^{4} a_{t} x(i, t)\right\|\right. & \\
& \left.+\sum_{i \in W}\left\|x(i)-\sum_{t=1}^{4} a_{t} y(i, t)\right\|\right\}
\end{aligned}
$$




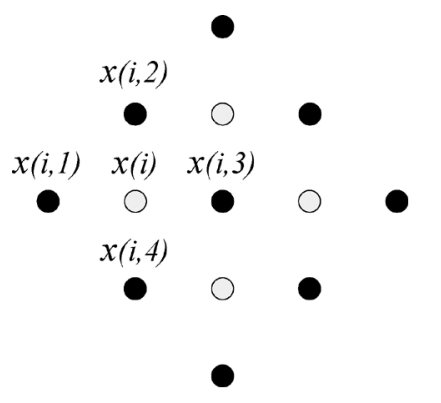

Fig. 5. Spatial configuration in second pass of PARM.

where $W$ is a small local window in which parameters $\boldsymbol{a}$ remain constant. The sample relationships involved in (1) is depicted in Fig. 4(b). The details on the merits of the PARM model and the solution of (1) can be found in [10].

Upon the completion of the first pass, the blue/red band becomes a quincunx lattice, being the same as the green band of Bayer CFA. The remaining missing blue/red pixels are to be interpolated in the second pass. The interpolation problem in the second pass is essentially the same as the one just discussed. The only difference is that we interpolate the missing pixels $y(i) \in I_{h}$ using their four 4-connected neighbors, which are either known in $I_{l}$ or estimated in the first pass. The problem has the same formulation of nonlinear optimization as in (1), if we simply rotate the spatial configuration of Fig. 4(a) by 45 degrees (see Fig. 5). Evidently, the intraband interpolation of the green band can be done in the same way. With the above two-step interpolation process, we obtain an estimated full color image by PARM model.

\section{Linear Minimum MEAN-SQUare Fusion OF InTRA- AND INTERBAND ESTIMATES}

As stated in the introduction, the intraband demosaicking by the PARM model is meant to be used in conjunction with an interband demosaicking method for more robust results. Any existing interband demosaicking method can be improved by the PARM intraband demosaicking technique.

For each missing sample $x(n)$, where $n$ denoting the pixel location, let its intraband and interband estimates be $\hat{x}_{1}(n)$ and $\hat{x}_{2}(n)$, respectively. The corresponding estimation errors are denoted by $\varepsilon_{1}(n)$ and $\varepsilon_{2}(n)$, namely

$$
\left\{\begin{array}{l}
x(n)=\hat{x}_{1}(n)+\varepsilon_{1}(n) \\
x(n)=\hat{x}_{2}(n)+\varepsilon_{2}(n)
\end{array} .\right.
$$

Our objective is to fuse $\hat{x}_{1}(n)$ and $\hat{x}_{2}(n)$ into a combined estimate which is more accurate. We employ the weighted average strategy and let the fused estimate be

$$
\hat{x}(n)=w_{1}(n) \hat{x}_{1}(n)+w_{2}(n) \hat{x}_{2}(n)
$$

with affine weighting factors $w_{1}(n)+w_{2}(n)=1$, such that $\hat{x}(n)$ is the linear minimum mean-square estimate of $x(n)$. The weights $w_{1}(n)$ and $w_{2}(n)$ are determined to minimize the meansquare error of $\hat{x}(n)$

$$
\sigma_{\hat{x}}^{2}(n)=E\left[(x(n)-\hat{x}(n))^{2}\right]
$$

or using (3)

$$
\begin{aligned}
\sigma_{\hat{x}}^{2}(n)=w_{1}^{2}(n) \sigma_{1}^{2}(n) & +w_{2}^{2}(n) \sigma_{2}^{2}(n) \\
& +2 \cdot w_{1}(n) w_{2}(n) \cdot E\left[\varepsilon_{1}(n) \varepsilon_{2}(n)\right]
\end{aligned}
$$

where $\sigma_{1}^{2}(n)$ and $\sigma_{2}^{2}(n)$ are the variances of $\varepsilon_{1}(n)$ and $\varepsilon_{2}(n)$ in a local window centered at pixel location $n$.

Noting that $w_{2}=1-w_{1}$

$$
\begin{aligned}
\sigma_{\hat{x}}^{2}(n)= & w_{1}^{2}(n) \cdot\left(\sigma_{1}^{2}(n)+\sigma_{2}^{2}(n)-2 E\left[\varepsilon_{1}(n) \varepsilon_{2}(n)\right]\right) \\
& +2 w_{1}(n) \cdot\left(-\sigma_{2}^{2}(n)+E\left[\varepsilon_{1}(n) \varepsilon_{2}(n)\right]\right)+\sigma_{2}^{2}(n) .
\end{aligned}
$$

To minimize $\sigma_{\hat{x}}^{2}(n)$, we set the partial differential of $\sigma_{\hat{x}}^{2}(n)$ with respect to $w_{1}(n)$ to zero

$$
\begin{array}{r}
\frac{\partial \sigma_{\hat{x}}^{2}(n)}{\partial w_{1}(n)}=2 w_{1}(n) \cdot\left(\sigma_{1}^{2}(n)+\sigma_{2}^{2}(n)-2 E\left[\varepsilon_{1}(n) \varepsilon_{2}(n)\right]\right) \\
-2 \sigma_{2}^{2}(n)+2 E\left[\varepsilon_{1}(n) \varepsilon_{2}(n)\right]=0
\end{array}
$$

which yields

$$
\begin{aligned}
& w_{1}(n)=\frac{\sigma_{2}^{2}(n)-E\left[\varepsilon_{1}(n) \varepsilon_{2}(n)\right]}{\sigma_{1}^{2}(n)+\sigma_{2}^{2}(n)-2 E\left[\varepsilon_{1}(n) \varepsilon_{2}(n)\right]} \\
& w_{2}(n)=\frac{\sigma_{1}^{2}(n)-E\left[\varepsilon_{1}(n) \varepsilon_{2}(n)\right]}{\sigma_{1}^{2}(n)+\sigma_{2}^{2}(n)-2 E\left[\varepsilon_{1}(n) \varepsilon_{2}(n)\right]} .
\end{aligned}
$$

Substituting (8) into (3) obtains $\hat{x}(n)$, the optimally weighted estimate of intra and interband demosaicking. The MSE of the optimal estimate is

$$
\sigma_{\hat{x}}^{2}(n)=\frac{\sigma_{1}^{2}(n) \cdot \sigma_{2}^{2}(n)-E^{2}\left[\varepsilon_{1}(n) \varepsilon_{2}(n)\right]}{\sigma_{1}^{2}(n)+\sigma_{2}^{2}(n)-2 E\left[\varepsilon_{1}(n) \varepsilon_{2}(n)\right]} .
$$

Obviously, $\sigma_{\hat{x}}^{2}(n)$ is less than either of $\sigma_{2}^{2}(n)$ and $\sigma_{1}^{2}(n)$.

The remaining task is to estimate $\sigma_{1}^{2}(n), \sigma_{2}^{2}(n)$ and $E\left[\varepsilon_{1}(n) \varepsilon_{2}(n)\right]$. For the PARM method, $\sigma_{1}^{2}(n)$ is simply the value of the objective function (1), which is a by-product of the nonlinear optimization process.

The estimation of $\sigma_{2}(n)$, the error variance of interband demosaicking, depends on the specific interband demosaicking method. Assuming the original image signal is stationary in a small window $W(n)$ centered at the missing sample $x(n)$, we estimate the demosaicking error $\varepsilon_{2}(n)$ of an interband method by interpolating the known samples $x(i)$ of CFA, $i \in W(n)$, from the neighboring estimated samples produced by the same interband demosaicking method. To be more concrete, let us examine how to estimate $\sigma_{2}(n)$ for the popular interband demosaicking method of Hamilton and Adams [6]. Using the above idea, the variance of $\varepsilon_{2}(n)$ is estimated by

$$
\sigma_{2}^{2}(n)=\frac{1}{N} \sum_{i \in W(n)}\left(x(i)-\hat{x}_{2}(i)\right)^{2}
$$

where $x(i) \in I_{l}$ are the known samples in the local window $W(n)$, and $\hat{x}_{2}(i)$ are the interpolation values generated by the interband method of [6], but using estimated samples $\hat{x}(n) \in I_{h}$ which are missing in the beginning. This uses the degree of fit of the directional interband filter of [6] in $W(n)$ to estimate $\sigma_{2}^{2}(n)$.

Consider an example depicted in Fig. 6, where the missing green sample at a blue sample position $B(0)$ in CFA. The error 


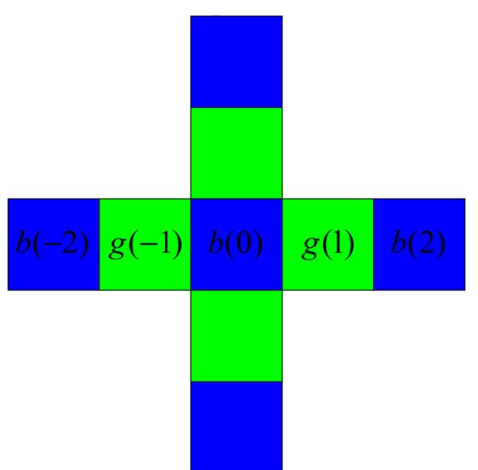

Fig. 6. Row and a column of mosaic data that intersect at a blue sampling position.

variance in estimating the missing green sample at this pixel is estimated by

$$
\sigma_{g}^{2}(0)=\frac{1}{N} \sum_{i \in W(0)}(g(i)-\hat{g}(i))^{2} .
$$

In the above summation $g(i)$ are the neighboring known green samples of CFA, and $\hat{g}(i)$ are the corresponding interpolation values of $g(i)$ as if they were to be estimated by the method of [6] in the same filtering direction as for $\hat{g}(0)$. Specifically, for the horizontal filtering direction, we have in (12) and (13)

$$
\begin{aligned}
& \hat{g}(+1)=\frac{\hat{g}(0)+\hat{g}(+2)}{2}+\frac{-b(0)+2 \hat{b}(+1)-b(+2)}{2} \\
& \hat{g}(-1)=\frac{\hat{g}(0)+\hat{g}(-2)}{2}+\frac{-b(0)+2 \hat{b}(-1)-b(-2)}{2} .
\end{aligned}
$$

This way we can estimate $\sigma_{g}^{2}(n)$ at a blue sample position in Bayer CFA. By the symmetry of CFA sample configuration, one can analogously derive the estimate of $\sigma_{g}^{2}(n)$ at the red sample position, and the estimates of $\sigma_{r}^{2}(n)$ and $\sigma_{b}^{2}(n)$.

Similarly, we can compute $E\left[\varepsilon_{1}(n) \varepsilon_{2}(n)\right]$

$$
\begin{aligned}
E\left[\varepsilon_{1}(n) \varepsilon_{2}(n)\right]=\frac{1}{N} \sum_{i \in W(n)}( & \left.(i)-\hat{x}_{2}(i)\right) \\
& \cdot\left(x(i)-\sum_{t=1}^{4} a_{t} y(i, t)\right) .
\end{aligned}
$$

\section{INTRA- AND INTERBAND ESTIMATION BY SUPPORT VECTOR REGRESSION}

In the proceeding section, the fusion of the intraband and interband estimates is treated and solved as a problem of linear minimum mean-square estimation. But this method can fail when the image signal exceeds the Nyquist frequency. In this case, the estimates of the missing color values are wrong but they still match the autoregressive model, and we grossly underestimate the error variance $\sigma$. To overcome this weakness, we take a machine learning approach to solve the problem of data fusion. Specifically, we use support vector regression (SVR) to find the optimal fusion weights $w_{1}$ and $w_{2}$.

Introduced by Vapnik [11], support vector machines (SVM) is a popular machine learning algorithm with the ability to per- form high-dimensional function estimation. Support vector regression (SVR) is a SVM-based regression technique. SVR operates in a feature space to approximate unknown functions in an output space, aiming to linearly estimate an unknown regression with nonlinear functions.

Suppose that the dependence of a scalar $d$ on a vector $\mathbf{z}$ can be described by a nonlinear regressive model

$$
d=f(\mathbf{z}) \text {. }
$$

To learn this model, we choose an appropriate training set consisting of input-output pairs

$$
\Omega=\left\{\left(\mathbf{z}_{1}, d_{1}\right),\left(\mathbf{z}_{2}, d_{2}\right), \ldots,\left(\mathbf{z}_{N}, d_{N}\right)\right\}
$$

where $\mathbf{z}_{i} \in \mathbb{R}^{d}$ is a sample value of the input vector $\mathbf{z}$ and has a target value $d_{i} \in \mathbb{R}$ that is the model output on $\mathbf{z}_{i}$. Our goal is to obtain an estimate of the function $f(\cdot): \mathbf{z} \rightarrow d$.

To this end, we postulate that an estimate of $d$, denoted by $r$, can be expanded in terms of a set of nonlinear basis functions $\left\{\varphi_{j}(\mathbf{z})\right\}_{j=0}^{m}$

$$
r=\sum_{j=0}^{m} \omega_{j} \varphi_{j}(\mathbf{z})=\omega^{T} \varphi(\mathbf{z})
$$

Now the estimation problem can be stated as one of minimizing the empirical risk

$$
R=\frac{1}{N} \sum_{i=1}^{N} L_{\epsilon}\left(d_{i}, r_{i}\right)
$$

subject to the inequality $\|\omega\|^{2} \leq c_{0}$, where $c_{0}$ is a constant, and $L_{\epsilon}\left(d_{i}, r_{i}\right)$ is a so-called $\epsilon$-loss function, which is defined below, to make the model estimate more robust

$$
L_{\epsilon}(d, r)= \begin{cases}|d-r|-\epsilon, & \text { for }|d-r| \geq \epsilon \\ 0, & \text { otherwise }\end{cases}
$$

with $\epsilon \geq 0$ being a prescribed parameter. This constrained optimization problem can be reformulated by introducing two sets of nonnegative slack variables $\left\{\xi_{i}\right\}_{i=1}^{N}$ and $\left\{\xi_{i}^{\prime}\right\}_{i=1}^{N}$

$$
\min \Phi\left(\omega, \xi_{i}, \xi_{i}^{\prime}\right)=C\left(\sum_{i=1}^{N}\left(\xi_{i}+\xi_{i}^{\prime}\right)\right)+\frac{1}{2} \omega^{T} \omega
$$

subject to

$$
\begin{aligned}
& d_{i}-\omega^{T} \varphi\left(\mathbf{x}_{i}\right) \leq \epsilon+\xi_{i} \\
& \omega^{T} \varphi\left(\mathbf{x}_{i}\right)-d_{i} \leq \epsilon+\xi_{i}^{\prime} \\
& \xi_{i}, \xi_{i}^{\prime} \geq 0, \quad \text { for } i=1,2, \cdots, N
\end{aligned}
$$

where $C$ is a user-specified constant. There are two inequalities to bound the output training for each $r_{i}$, regulated by corresponding slack variables $\xi_{i}$ and $\xi_{i}^{\prime}$. The effect is that the estimate $r$ can deviate for a prespecified error and cost. 
The constrained optimization problem of (20) can be solved in a Lagrangian form as the following dual maximization problem:

$$
\begin{gathered}
\max \quad Q\left(\alpha_{i}, \alpha_{i}^{\prime}\right)=\sum_{i=1}^{N} d_{i}\left(\alpha_{i}-\alpha_{i}^{\prime}\right)-\epsilon \sum_{i=1}^{N}\left(\alpha_{i}+\alpha_{i}^{\prime}\right) \\
-\frac{1}{2} \sum_{i=1}^{N} \sum_{j=1}^{N}\left(\alpha_{i}-\alpha_{i}^{\prime}\right)\left(\alpha_{j}-\alpha_{j}^{\prime}\right) K\left(\mathbf{z}, \mathbf{z}_{i}\right)
\end{gathered}
$$

subject to

$$
\begin{aligned}
& \sum_{i=1}^{N}\left(\alpha_{i}-\alpha_{i}^{\prime}\right)=0 \\
& 0 \leq \alpha_{i}, \alpha_{i}^{\prime} \leq C, \quad i=1,2, \cdots, N
\end{aligned}
$$

where $\alpha_{i}, \alpha_{i}^{\prime}$ are the Lagrange multipliers, and $K\left(\mathbf{z}_{i}, \mathbf{z}_{j}\right)=$ $\varphi\left(\mathbf{z}_{i}\right)^{T} \varphi\left(\mathbf{z}_{j}\right)$ is called the inner-product kennel. A significance of (21) is that the dual problem is cast entirely in terms of the training data. As a result, the objective function $Q\left(\alpha_{i}, \alpha_{i}^{\prime}\right)$ depends only on the input patterns in the form of inner-product kennel. Most importantly, one may use the inner-product kennel $K\left(\mathbf{z}, \mathbf{z}_{i}\right)$ to construct the minimum-risk discriminating hyperplane in the feature space without having to explicitly work with the feature space itself. The estimate can now be rewritten as

$$
r=\omega^{T} \varphi(\mathbf{z})=\sum_{i=1}^{N}\left(\alpha_{i}-\alpha_{i}^{\prime}\right) K\left(\mathbf{z}, \mathbf{z}_{i}\right) .
$$

Thus, given a feature $\mathbf{z}$, we will use (22) to estimate $r$ as learnt from the training set.

In our demosaicking problem, SVR is used to find the optimal fusion weights $w_{1}$ and $w_{2}$ at each pixel position. Considering $w_{1}+w_{2}=1$, we just estimate one of them. In the nonlinear regressive model the dependence of $w_{1}$ on a feature vector $\mathbf{z}$ is described by

$$
w_{1}=f(\mathbf{z}) .
$$

The remaining task is to select suitable feature vector $\mathbf{z}$ and a training set $\Omega$ by which SVR can provide a good estimate of the function $f(\cdot): \mathbf{z} \rightarrow w_{1}$. In the training process, we know the real error terms $\epsilon_{1}(n)$ and $\epsilon_{2}(n)$ and, hence, can get the ideal weight $w_{1}$ using (8).

The most critical factor in designing a good learning machine for color demosaicking is the selection of suitable features that can indicate which demosaicking method is better for the current pixel. Besides $\sigma_{1}$ and $\sigma_{2}$, we need to furnish SVR with more statistically significant features. The sample variance $\operatorname{Var}(x)$ is a useful feature. The other features are the interband linear correlation coefficients

$$
\rho_{x, y}=\frac{\operatorname{Cov}(x, y)}{\sqrt{\operatorname{Var}(x) \operatorname{Var}(y)}}
$$

where $x, y$ are samples of two different primary bands (i.e., $\mathrm{R}, \mathrm{G}, \mathrm{B})$ in a local window $W(n)$ centered at the missing sample $x(n)$. This gives us three features $\rho=\left(\rho_{R G}, \rho_{G B}, \rho_{R B}\right)$.

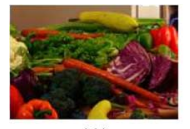

(1)

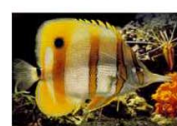

(5)

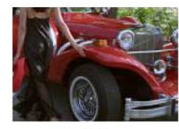

(9)

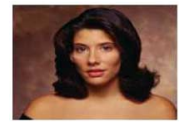

(13)

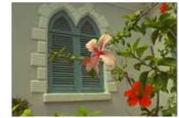

(17)

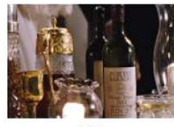

(2)

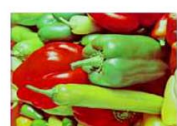

(6)

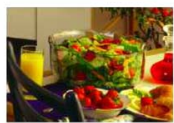

(10)

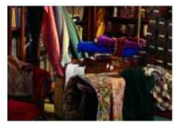

(14)

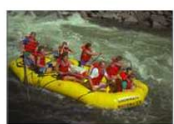

(18)

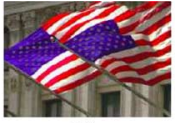

(3)

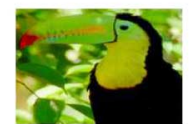

(7)

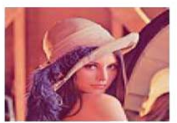

(11)

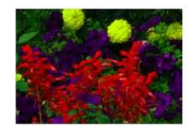

(15)

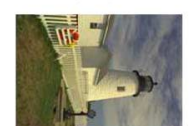

(19)

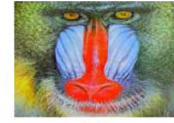

(4)

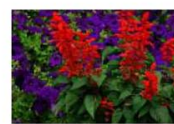

(8)

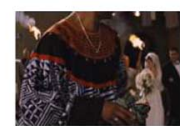

(12)

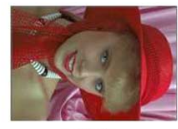

(16)

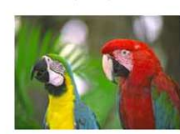

(20)
Fig. 7. Test images used in this paper.

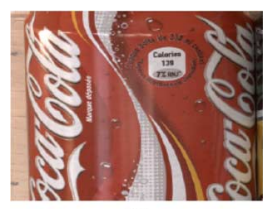

(1)

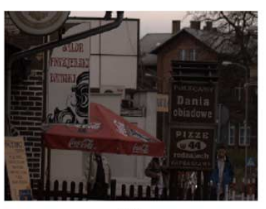

(2)

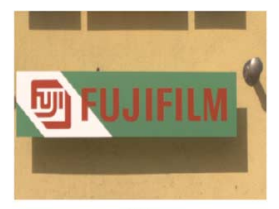

(3)
Fig. 8. Raw CFA data used in this paper (after demosaicking as thumbnail here).

Due to the fact that an interband demosaicking method heavily relies on spectral correlation, the other important feature is the saturation level of the color

$$
S=1-\frac{3}{r(n)+g(n)+b(n)} \min (r(n), g(n), b(n)) .
$$

By convention, we let $S=1$ if $r(n)=g(n)=b(n)=0$. Finally, we get a richer feature vector

$$
\mathbf{z}=\left\{\sigma_{1}, \sigma_{2}, \rho, \operatorname{Var}, S\right\}
$$

The selection of a suitable training set is also important for a learning algorithm [12], particularly so if our goal is to achieve best possible visual quality rather than just high PSNR. If the training set is drawn at random and uniformly from representative images independent of image features, then the population of the training set will be dominated by samples drawn from relatively smooth areas, because natural images mostly consist of large smoothly shaded regions. These samples will overwhelmingly influence the SVR design because of their sheer weight. On the other hand, the human visual system is very sensitive to edges and line textures. even though they present only a minority of the sample population. Therefore, one should design the SVR using samples drawn from the activity regions of the images. 
TABLE II

PSNR (dB) RESULTS OF DIFFERENT METHODS

\begin{tabular}{|c|c|c|c|c|c|c|c|c|c|c|c|c|}
\hline Image & \multicolumn{3}{|c|}{$\overline{\text { Fig.7(1) }}$} & \multicolumn{3}{|c|}{$\overline{~ F i g .7(2) ~}$} & \multicolumn{3}{|c|}{ Fig.7(3) } & \multicolumn{3}{|c|}{ Fig.7(4) } \\
\hline channels & $\mathbf{R}$ & G & B & $\mathbf{R}$ & G & B & $\mathbf{R}$ & G & B & $\mathbf{R}$ & G & B \\
\hline Gunturk et al.'s method [2] & 34.36 & 36.46 & 34.87 & 32.40 & 34.17 & 28.85 & 30.26 & 32.12 & 29.69 & 24.92 & 27.67 & 24.34 \\
\hline Adaptive homogeneity [3] & 34.52 & 38.71 & 34.61 & 33.37 & 35.81 & 28.82 & 30.44 & 34.52 & 29.66 & 24.70 & 27.86 & 24.19 \\
\hline Hamilton-Adams method [6] & 36.22 & 40.03 & 36.02 & 34.43 & 36.98 & 29.19 & 31.59 & 35.37 & 30.63 & 24.03 & 26.91 & 24.26 \\
\hline PCSD [5] & 36.17 & 40.15 & 36.09 & 34.20 & 36.62 & 29.29 & 31.40 & 35.30 & 30.73 & 25.50 & 28.26 & 25.07 \\
\hline EUSSC [8] & 36.04 & 38.88 & 36.06 & 33.17 & 35.31 & 29.10 & 31.10 & 33.45 & 30.28 & 25.43 & 28.30 & 24.91 \\
\hline bicubic and method [6] fused & 37.42 & 39.81 & 36.77 & 35.31 & 37.80 & 28.82 & 32.05 & 34.86 & 30.88 & 24.18 & 26.34 & 24.04 \\
\hline NEDI [14] and method [6] fused & 36.89 & 40.65 & 36.8 & 34.72 & 39.11 & 28.75 & 32.63 & 36.85 & 31.47 & 25.61 & 27.89 & 24.61 \\
\hline Proposed(LMMSE) & 37.69 & 41.03 & 37.24 & 34.84 & 39.44 & 29.02 & 32.24 & 37.17 & 31.33 & 24.32 & 27.65 & 24.39 \\
\hline Proposed(SVR) & 37.85 & 41.21 & 37.42 & 35.42 & 39.31 & 29.14 & 32.74 & 37.11 & 31.67 & 24.62 & 27.59 & 24.65 \\
\hline Image & \multicolumn{3}{|c|}{ Fig.7(5) } & \multicolumn{3}{|c|}{ Fig.7(6) } & \multicolumn{3}{|c|}{ Fig.7(7) } & \multicolumn{3}{|c|}{ Fig.7(8) } \\
\hline channels & $\mathbf{R}$ & G & B & $\mathbf{R}$ & G & B & $\mathbf{R}$ & G & B & $\mathbf{R}$ & & B \\
\hline Gunturk et al.'s method & 26.73 & 29.44 & 26.55 & 29.66 & 33.24 & 31.18 & 42.15 & 43.61 & 41.59 & 28.29 & 30.04 & 27.91 \\
\hline Adaptive homogeneity & 26.58 & 29.30 & 26.23 & 27.88 & 34.06 & 27.31 & 41.84 & 44.17 & 39.82 & 27.94 & 32.70 & 27.19 \\
\hline Hamilton-Adams method & 26.82 & 29.20 & 26.60 & 29.13 & 34.06 & 29.58 & 42.79 & 45.57 & 42.03 & 30.70 & 35.60 & 29.74 \\
\hline PCSD & 27.40 & 29.96 & 27.00 & 30.65 & 34.66 & 31.75 & 43.71 & 46.22 & 42.33 & 30.35 & 34.79 & 29.43 \\
\hline EUSSC & 27.08 & 29.78 & 26.84 & 31.40 & 34.60 & 31.94 & 42.84 & 45.35 & 41.03 & 30.18 & 33.17 & 29.85 \\
\hline bicubic and method [6] fused & 26.81 & 29.05 & 27.16 & 29.63 & 33.85 & 29.84 & 41.98 & 44.77 & 43.07 & 33.42 & 36.83 & 32.19 \\
\hline NEDI and method [6] fused & 26.93 & 29.38 & 27.39 & 30.57 & 34.62 & 31.13 & 42.09 & 46.19 & 42.88 & 32.44 & 37.38 & 31.88 \\
\hline Proposed(LMMSE) & 27.00 & 29.30 & 27.24 & 29.78 & 34.23 & 29.91 & 42.55 & 46.21 & 42.54 & 33.53 & 38.40 & 32.59 \\
\hline Proposed(SVR) & 27.19 & 29.46 & 27.41 & 29.85 & 34.30 & 30.01 & 42.50 & 46.36 & 42.84 & & or traini & \\
\hline Image & \multicolumn{3}{|c|}{ Fig.7(9) } & \multicolumn{3}{|c|}{ Fig.7(10) } & \multicolumn{3}{|c|}{ Fig.7(11) } & \multicolumn{3}{|c|}{ Fig.7(12) } \\
\hline channels & $\mathbf{R}$ & G & B & $\mathbf{R}$ & G & B & $\mathbf{R}$ & G & B & $\mathbf{R}$ & G & B \\
\hline Gunturk et al.'s method & 34.04 & 36.78 & 32.58 & 32.53 & 34.98 & 33.05 & 33.49 & 36.06 & 32.85 & 34.55 & 36.69 & 31.48 \\
\hline Adaptive homogeneity & 34.59 & 39.53 & 33.36 & 32.85 & 38.06 & 33.16 & 33.23 & 36.78 & 32.69 & 35.24 & 38.11 & 31.55 \\
\hline Hamilton-Adams' method & 36.66 & 40.56 & 34.57 & 34.79 & 39.73 & 35.07 & 34.28 & 37.09 & 32.88 & 36.31 & 39.22 & 31.85 \\
\hline PCSD & 36.03 & 40.35 & 34.61 & 34.47 & 39.39 & 34.69 & 34.34 & 37.49 & 33.17 & 36.05 & 38.72 & 31.90 \\
\hline EUSSC & 36.30 & 39.40 & 34.28 & 34.12 & 37.79 & 34.64 & 34.01 & 36.89 & 33.12 & 35.10 & 37.58 & 31.80 \\
\hline bicubic and method [6] fused & 39.34 & 39.96 & 33.60 & 36.25 & 39.56 & 36.57 & 36.73 & 36.75 & 32.42 & 37.66 & 40.59 & 31.80 \\
\hline NEDI and method [6] fused & 38.44 & 42.29 & 32.94 & 35.58 & 40.81 & 36.84 & 36.79 & 37.34 & 32.9 & 37.16 & 41.48 & 31.86 \\
\hline Proposed(LMMSE) & 38.94 & 42.59 & 34.21 & 36.31 & 41.40 & 37.06 & 36.81 & 37.53 & 32.80 & 37.25 & 41.71 & 32.00 \\
\hline Proposed(SVR) & \multicolumn{3}{|c|}{ For training } & \multicolumn{3}{|c|}{ For training } & \multirow{2}{*}{\multicolumn{3}{|c|}{ For training }} & & or traini & \\
\hline Image & & Fig.7(13 & & & Fig.7(1 & & & & & & & \\
\hline channels & $\mathbf{R}$ & G & B & $\mathbf{R}$ & G & B & $\mathbf{R}$ & G & B & & & \\
\hline Gunturk et al.'s method & 38.62 & 40.84 & 37.62 & 30.33 & 33.42 & 30.00 & 29.85 & 32.47 & 30.83 & & & \\
\hline Adaptive homogeneity & 38.52 & 41.53 & 37.46 & 30.33 & 35.30 & 29.83 & 29.54 & 35.08 & 30.31 & & & \\
\hline Hamilton-Adams method & 38.94 & 41.90 & 37.45 & 31.74 & 36.18 & 31.01 & 32.47 & 37.83 & 32.82 & & & \\
\hline PCSD & 39.15 & 42.07 & 37.98 & 31.79 & 36.31 & 31.06 & 32.01 & 37.09 & 32.43 & & & \\
\hline EUSSC & 39.08 & 41.72 & 37.97 & 31.19 & 34.95 & 30.74 & 31.49 & 35.42 & 32.61 & & & \\
\hline bicubic and method [6] fused & 38.98 & 42.27 & 37.64 & 32.07 & 35.43 & 30.96 & 35.12 & 38.81 & 35.36 & & & \\
\hline NEDI and me & 39.5 & 43.17 & 38.05 & 31.87 & 36.8 & 31.08 & 33.91 & 39.45 & 35.14 & & & \\
\hline Proposed(LMMSE) & 39.39 & 42.89 & 37.75 & 32.03 & 37.10 & 31.27 & 35.25 & 40.28 & 35.90 & & & \\
\hline Proposed(SVR) & & or traini & & & or train & & & or traini & & & & \\
\hline Image & & erage(1) & & & Averag & & & & & & & \\
\hline channels & $\mathbf{R}$ & G & B & $\mathbf{R}$ & G & B & & & & & & \\
\hline Gunturk et & 31.50 & 33.82 & 31.01 & 32.15 & 34.53 & 31.56 & & & & & & \\
\hline Adaptive $\mathrm{h}$ & 31.33 & 34.92 & 30.09 & 32.10 & 36.10 & 31.08 & & & & & & \\
\hline Hamilton-Adams method & 32.14 & 35.45 & 31.19 & 33.39 & 37 & 32.25 & & & & & & \\
\hline PCSD & 32.72 & 35.88 & 31.75 & 33.55 & 37.16 & 32.50 & & & & & & \\
\hline EUSSC & 32.44 & 35.10 & 31.45 & 33.24 & 36.17 & 32.34 & & & & & & \\
\hline bicubic and method [6] fused & 32.48 & 35.21 & 31.51 & 34.46 & 37.11 & 32.74 & & & & & & \\
\hline NEDI and met & 32.68 & 36.38 & 31.86 & 34.30 & 38.23 & 32.91 & & & & & & \\
\hline Proposed (LMMSE) & 32.63 & 36.43 & 31.67 & 34.53 & 38.46 & 33.02 & & & & & & \\
\hline roposed (SVR) & 32.88 & 36.48 & 31.88 & 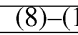 & 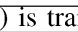 & & & & & & & \\
\hline
\end{tabular}

This design will not affect the visual quality of the smooth regions, for which the two estimates $\hat{x}_{1}$ and $\hat{x}_{2}$ will be close to each other anyways and either is a good estimate.

The first selection criterion for the training set is

$$
\left|\hat{x}_{1}-\hat{x}_{2}\right|>\Delta
$$

where $\Delta$ is a threshold. This is because when the two estimates $x_{1}$ and $x_{2}$ differ significantly from each other, it becomes critical to give lager weight to the one that is closer to the missing sample. The penalty of get a wrong fusion coefficient is potentially high. The second selection criterion for the training set is

$$
|| x-\hat{x}_{1}\left|-\sigma_{1}\right|<\eta \quad \text { and } \quad|| x-\hat{x}_{2}\left|-\sigma_{2}\right|<\eta
$$

where $\eta$ is also a threshold. The intent is to exclude the samples for which the errors of intraband and interband demosaicking $\sigma_{1}$ and $\sigma_{2}$ are estimated incorrectly. The two selection criteria make the training set more relevant and robust.
In our experiments, we used the SVR algorithm implemented by the software package libsvm [13] and chose the thresholds $\Delta=5, \eta=8$ in (27) and (28), respectively. The radial basis function (RBF) is employed as the inner kernel function of SVR

$$
K\left(\mathbf{x}, \mathbf{x}_{i}\right)=\exp \left(-\gamma\left\|\mathbf{x}-\mathbf{x}_{i}\right\|^{2}\right)
$$

where $\gamma$ is a user-specified constant, the same as the parameters $\epsilon$ and $C$ in SVR. These three parameters are selected by experiment using grid-search and cross-validation to find the best values, so that the SVR can accurately predict unknown data. We finally find $\gamma=0.1, C=1$ and $\epsilon=0.1$. More details about the selection of the three parameters can be found in [13].

\section{EXPERIMENTAL RESULTS AND REMARKS}

The proposed hybrid color demosaicking algorithm is implemented in both LMMSE and SVR variants, and compared with five other methods: the popular method of Hamilton and 
TABLE III

PSNR (dB) RESULTS OF DIFFERENT METHODS ON KADAK SET

\begin{tabular}{|c|c|c|c|c|c|c|c|c|c|}
\hline Image & \multicolumn{3}{|c|}{ Fig.7(16) } & \multicolumn{3}{|c|}{ Fig.7(17) } & \multicolumn{3}{|c|}{ Fig.7(18) } \\
\hline channels & $\mathbf{R}$ & $\mathbf{G}$ & B & $\mathbf{R}$ & G & $\mathbf{B}$ & $\mathbf{R}$ & G & B \\
\hline Gunturk et al.'s method & 39.52 & 40.58 & 40.06 & 42.48 & 43.64 & 39.95 & 36.07 & 37.72 & 34.32 \\
\hline Adaptive homogeneity & 36.59 & 41.19 & 40.50 & 40.02 & 42.63 & 39.36 & 33.92 & 37.82 & 34.72 \\
\hline Hamilton-Adams' method & 37.61 & 40.66 & 39.56 & 40.65 & 42.17 & 39.92 & 35.01 & 37.29 & 35.19 \\
\hline PCSD & 38.54 & 42.58 & 41.52 & 41.18 & 43.36 & 40.32 & 35.91 & 39.08 & 36.17 \\
\hline EUSSC & 38.60 & 43.40 & 42.18 & 42.30 & 45.54 & 41.62 & 35.94 & 40.39 & 36.30 \\
\hline bicubic and method [6] fused & 36.45 & 39.08 & 37.10 & 37.69 & 39.80 & 37.46 & 32.41 & 34.77 & 32.62 \\
\hline NEDI and method [6] fused & 36.06 & 40.09 & 37.07 & 37.55 & 41.62 & 37.61 & 32.35 & 36.25 & 32.87 \\
\hline Proposed (LMMSE) & 36.54 & 40.09 & 38.00 & 39.03 & 41.57 & 38.61 & 33.20 & 36.37 & 33.36 \\
\hline Image & \multicolumn{3}{|c|}{ Fig.7(19) } & \multicolumn{3}{|c|}{ Fig.7(20) } & \multicolumn{3}{|c|}{ Average } \\
\hline channels & $\mathbf{R}$ & G & $\mathbf{B}$ & $\mathbf{R}$ & G & $\mathbf{B}$ & $\mathbf{R}$ & $\mathbf{G}$ & $\mathbf{B}$ \\
\hline Gunturk et al.'s method & 39.33 & 42.54 & 38.61 & 42.69 & 43.65 & 40.09 & 40.02 & 41.63 & 38.61 \\
\hline Adaptive homogeneity & 38.00 & 39.64 & 37.52 & 40.33 & 43.92 & 41.15 & 37.77 & 41.04 & 38.65 \\
\hline Hamilton-Adams' method & 36.98 & 38.41 & 36.76 & 41.14 & 43.45 & 41.63 & 38.28 & 40.40 & 38.61 \\
\hline PCSD & 38.83 & 40.71 & 38.53 & 41.48 & 44.73 & 42.33 & 39.19 & 42.09 & 39.77 \\
\hline EUSSC & 39.40 & 42.87 & 39.21 & 41.89 & 45.64 & 42.50 & 39.63 & 43.57 & 40.36 \\
\hline bicubic and method [6] fused & 31.86 & 34.28 & 31.83 & 39.22 & 41.42 & 39.11 & 35.53 & 37.87 & 35.62 \\
\hline NEDI and method [6] fused & 31.60 & 37.75 & 31.77 & 39.30 & 42.59 & 39.04 & 35.37 & 39.66 & $\mathbf{3 5 . 6 7}$ \\
\hline Proposed (LMMSE) & 33.16 & 37.69 & 33.74 & 40.17 & 43.07 & 40.67 & 36.42 & 39.76 & 36.88 \\
\hline
\end{tabular}

Adams [6], the wavelet-based method of Gunturk et al. [2], the adaptive homogeneity method of Hirakawa and Parks [3], the primary-consistent soft-decision demosaicking (PCSD) method [5], and the EUSSC method of Chang and Tan [8]. To evaluate the impact of different intraband interpolation algorithms in the proposed hybrid demosaicking framework, we also include in the comparison group two different combinations: a) bicubic interpolator and Hamilton-Adam's method, and b) the NEDI interpolator [14] and Hamilton-Adam's method.

We use two sets of color images in our comparison study. The first set consists of 20 test color images that have a wide range of spectral correlations. These 20 images, listed in Fig. 7, are chosen from the test sets of JPEG, MPEG, SMPTE and as well as from the Kodak test set [Fig. 7(16)-(20)]. They are originally fully sampled RGB images and the corresponding mosaic images are simulated by down-sampling the test images with the Bayer CFA pattern. For the said simulated CFA images we can compare the different demosaicking methods in PSNR since the underlying fully sampled color images are known. The second test set consists of three raw CFA images taken by Canon digital cameras, listed in Fig. 8, which are downloaded from [15]. This website provides these raw CFA images for the very purpose of evaluating the performance of different digital cameras. The second test image set allows us to compare the visual quality of different demosaicking methods in real action, although we cannot measure PSNR in this case. For the SVR variant of the new method, we include Fig. 7(8)-(15) in the training set and use the other images for testing.

We report in Table II and Table III the PSNR results of the eight demosaicking methods for the new general test set and the Kodak set, respectively. It can be seen from Table II that the tested methods are ranked from high to low in PSNR are 1) the proposed hybrid methods, 2) the PCSD method, 3) Hamilton-Adams' method, 4) the EUSSC method 5) adaptive homogeneity method, and 6) Gunturk et al.'s method. The new method of LMMSE variant gains on average $0.98 \mathrm{~dB}$ in red band, $1.3 \mathrm{~dB}$ in green band, and $0.52 \mathrm{~dB}$ in blue band over the PCSD method, which are substantial. We call reader's attention to the fact that the simple Hamilton-Adams' method actually

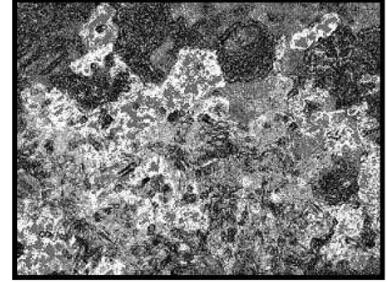

(a)

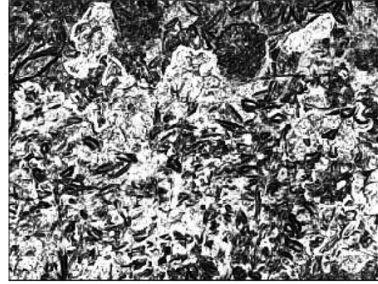

(b)
Fig. 9. (a) Map of estimated weight $w_{2}$ versus (b) the map of interband correlation of test image Fig. 7(15).

performs significantly better than the methods [2], [3], [8] on the general test set, exposing the sensitivity of these methods to spectral correlation. The average gain of the proposed method over the method [2] can be as much as $3.94 \mathrm{~dB}$ in the green band. On the other hand, the performance difference between the two variants of the new method is quite small. The SVR variant is about $0.17 \mathrm{~dB}$ better on average than the LMMSE variant.

However, on the Kodak images of exceptionally high spectral correlation, the PSNR ranking of the eight methods is very different. In Table III, Gunturk et al.'s method now achieves the third highest PSNR, whereas its ranking is the lowest in Table II. Only on the Kodak set Hamilton-Adams' method performs much worse than others. The sharp contrast between Table II and Table III demonstrates the necessity of using more balanced test images when evaluating different demosaicking techniques.

Given the large PSNR gains of the proposed methods over the existing demosaicking methods in Table II, one should expect clearly superior visual quality of the former. Figs. 11-17 are sample parts of seven original and reconstructed images by different evaluated methods. Of particular importance are the results of demosaicking the real raw CFA data, presented in Figs. 11-13. These results of real camera data suggest how robust different demosaicking algorithms are in reality in challenging cases. It can be seen that the new methods remove most of the objectionable color artifacts produced by the existing 


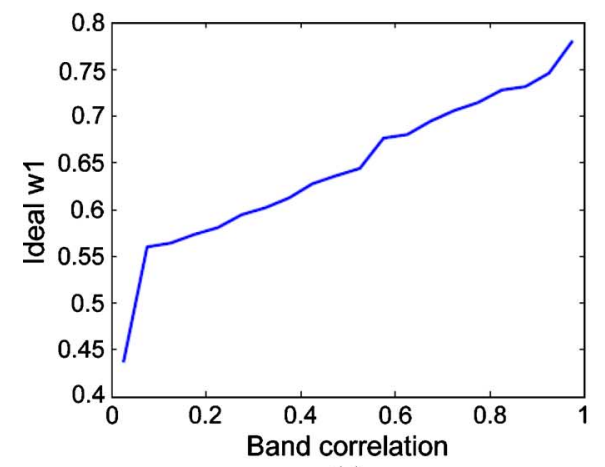

(a)

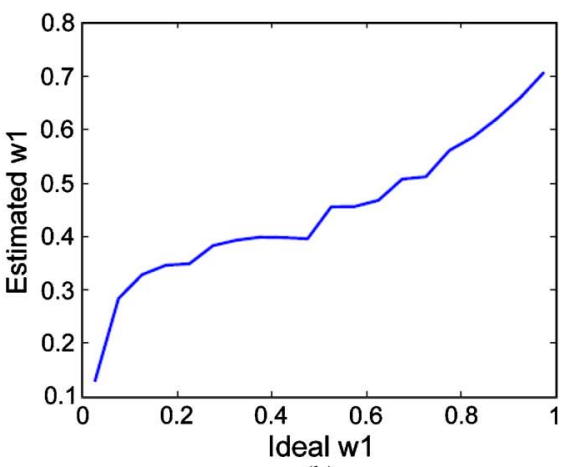

(b)

Fig. 10. (a) Ideal weight $w_{1}$ versus the interband correlation for test image Fig. 7(15). (b) Ideal $w_{1}$ versus estimated $w_{1}$ for test image Fig. 7(15).

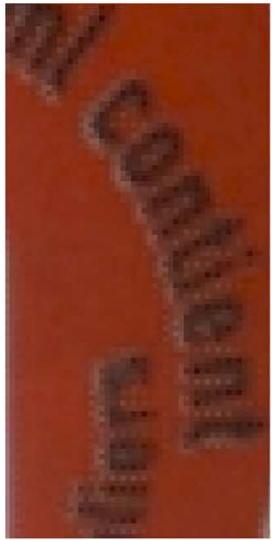

(a)

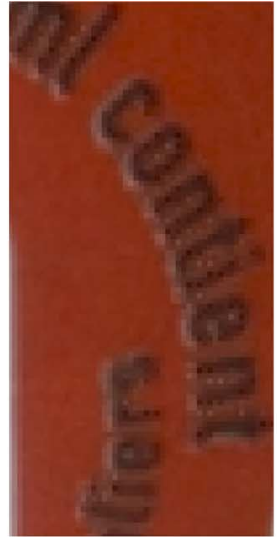

(b)

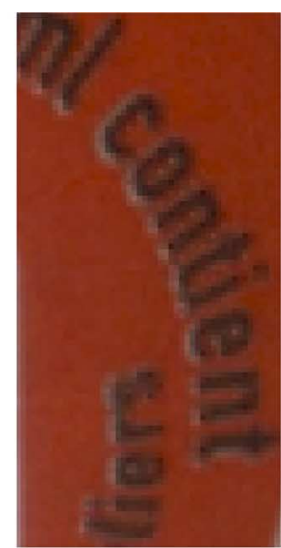

(c)

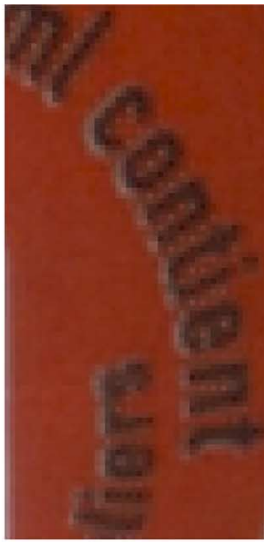

(d)

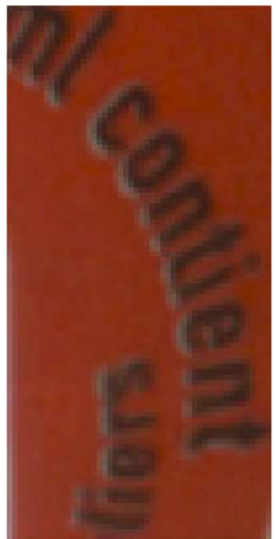

(e)

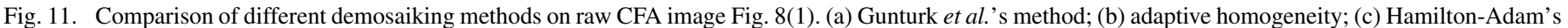
method; (d) PCSD; (e) proposed (LMMSE).

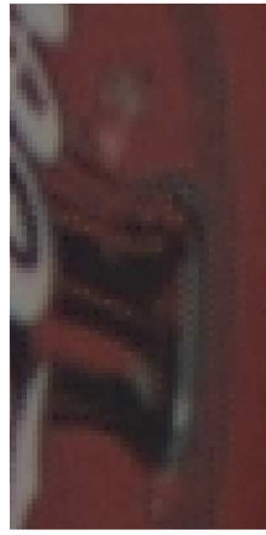

(a)

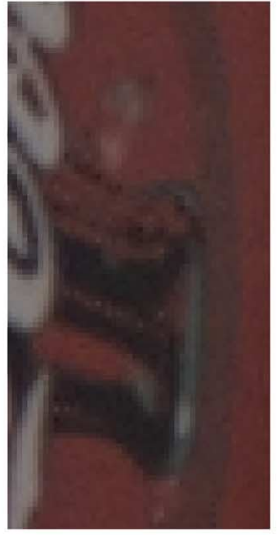

(b)

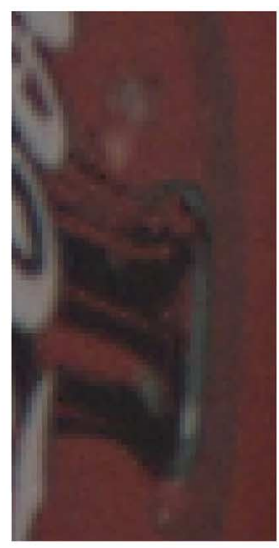

(c)

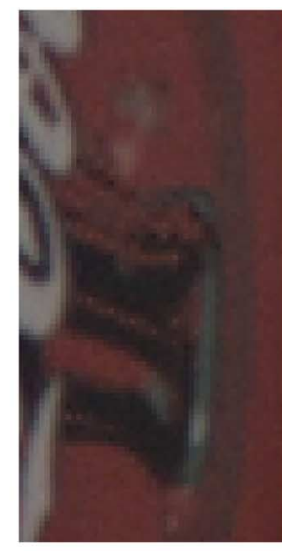

(d)

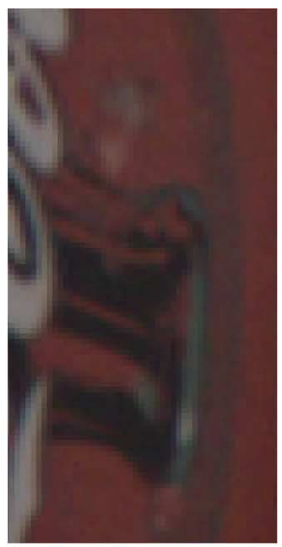

(e)

Fig. 12. Comparison of different demosaiking methods on raw CFA image Fig. 8(2). (a) Gunturk et al.'s method; (b) adaptive homogeneity; (c) Hamilton-Adam's method; (d) PCSD; (e) proposed (LMMSE).

interband demosaicking methods. These artifacts are highly objectionable to viewers because they are correlated with object boundaries and edges. Among the existing demosaicking methods, the Gunturk et al.'s method is the most susceptible to zipper effect, while the classic Hamilton-Adams' method appears to be the least susceptible.

Fig. 17 demonstrates that some of existing interband demosaicking methods are prone to artifacts even on colors of low saturation, when being subject to sensor noises. Note that sensor noises can distort the spectral correlation and, hence, derail the interband demosaicking methods. The new methods do not magnify sensor noises as the Hamilton-Adams' method and Gunturk et al.'s method.

To demonstrate the adaptability of the proposed method to varying spectral correlations, we present in Fig. 9, as an example, the intensity map of the weight $w_{2}$ in (8) in comparison with the interband correlation map for image Fig. 7(15). To quantify this adaptability we plot in Fig. 10 the curve of ideal weight $w_{1}$ as a function of interband correlation. Also, to demonstrate the effectiveness of the proposed fusion method, the curve of the ideal weight $w_{1}$ versus the estimated value of $w_{1}$ is included in Fig. 10, as well. 


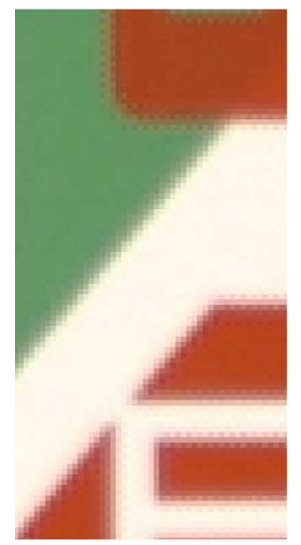

(a)

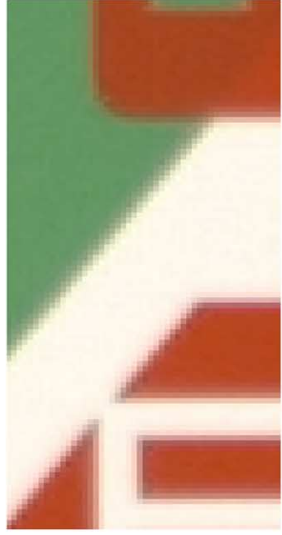

(b)

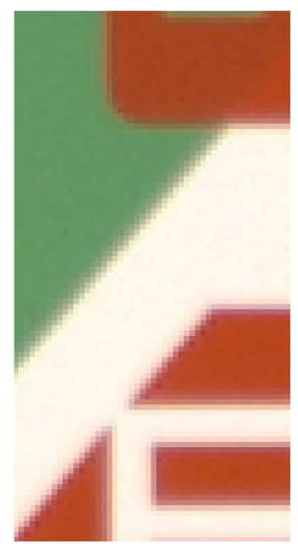

(c)

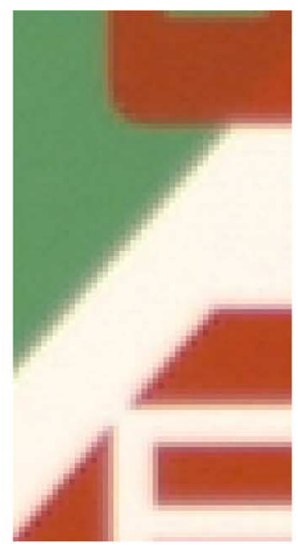

(d)

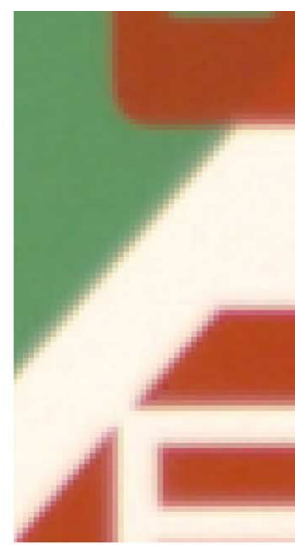

(e)

Fig. 13. Comparison of different demosaiking methods on raw CFA image Fig. 8(3). (a) Gunturk et al.'s method; (b) adaptive homogeneity; (c) Hamilton-Adam's method; (d) PCSD; (e) proposed (LMMSE).

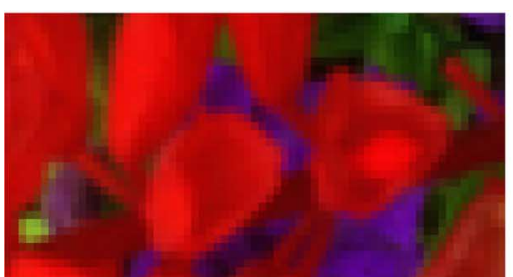

(a)

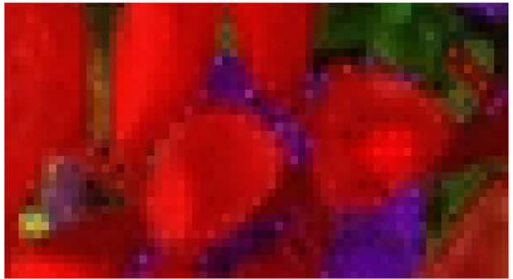

(d)

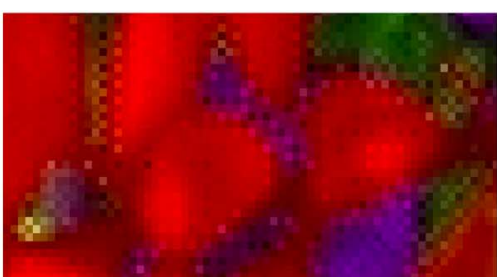

(b)

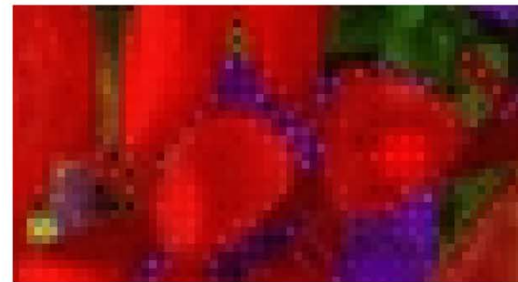

(e)

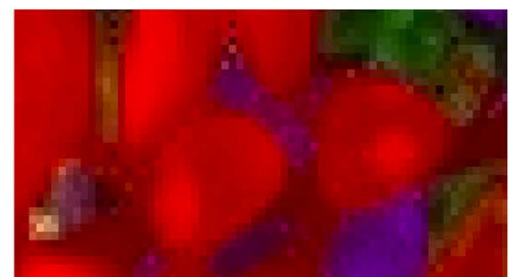

(c)

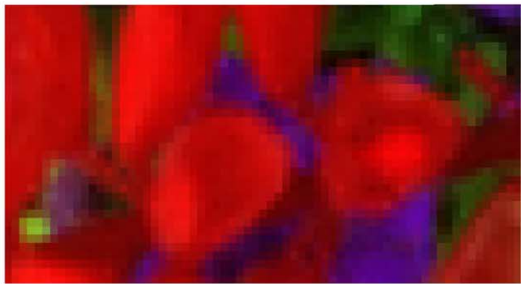

(f)

Fig. 14. Parts of the original and reconstructed images of Fig. 7(8). (a) Original; (b) Gunturk et al.'s method [2]; (c) adaptive homogeneity [3]; (d) HamiltonAdams' method [6]; (e) PCSD [5]; (f) proposed (LMMSE).

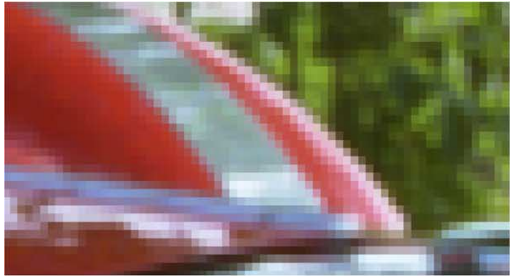

(a)

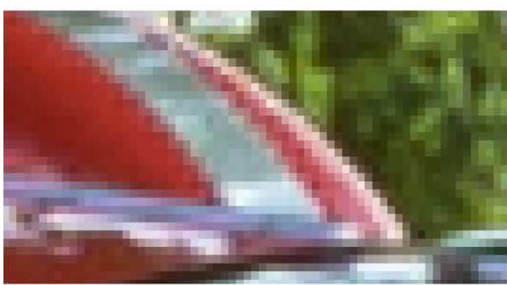

(d)

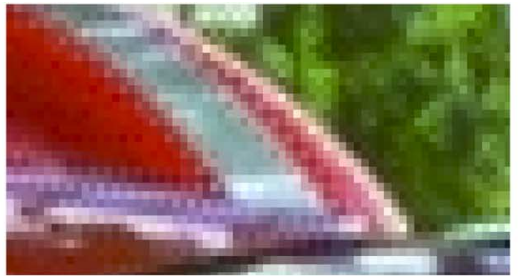

(b)

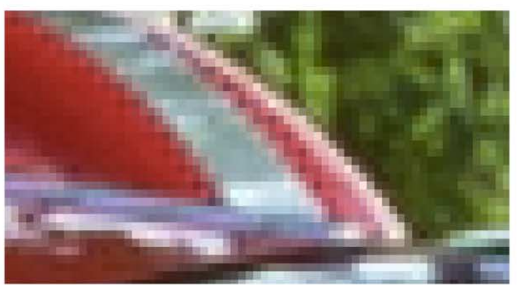

(e)

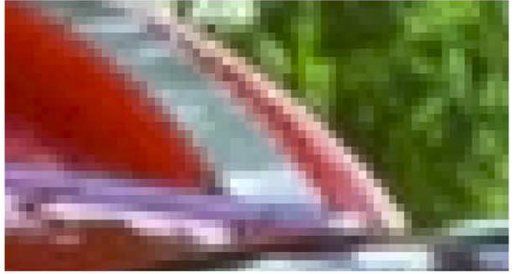

(c)

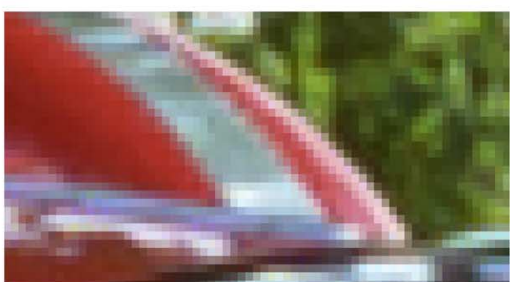

(f)

Fig. 15. Parts of the original and reconstructed images of Fig. 7(9). (a) Original; (b) Gunturk et al.'s method [2]; (c) adaptive homogeneity [3]; (d) HamiltonAdams' method [6]; (e) PCSD [5]; (f) proposed (LMMSE).

Also, we note that the LMMSE fusing of the bicubic interpolation result and Hamilton-Adams' demosaicking result is competitive against previous methods, but inferior to the proposed
LMMSE method that employs the PARM-based adaptive interpolation by an appreciable margin. Replacing the bicubic interpolator by the NEDI interpolator in the combination can expect 


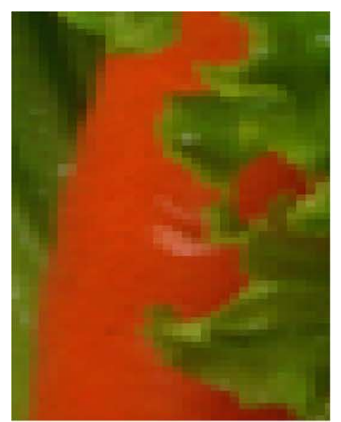

(a)

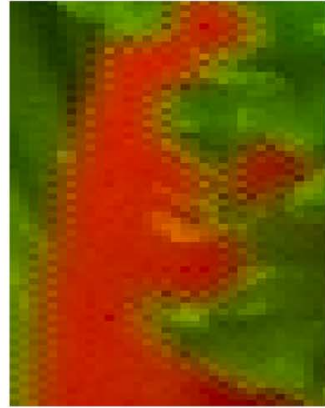

(b)

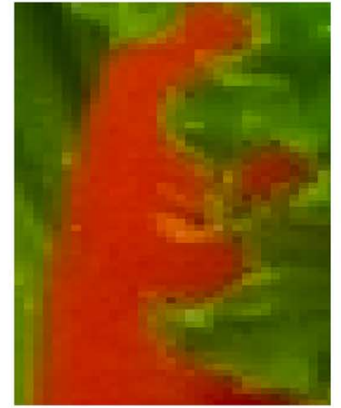

(c)

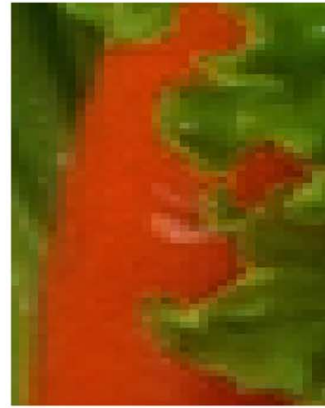

(d)

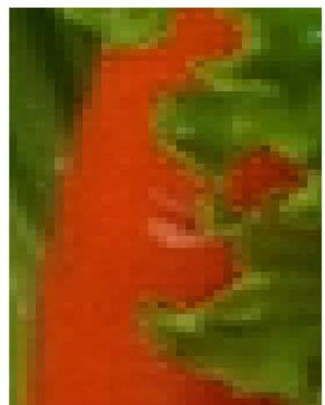

(e)

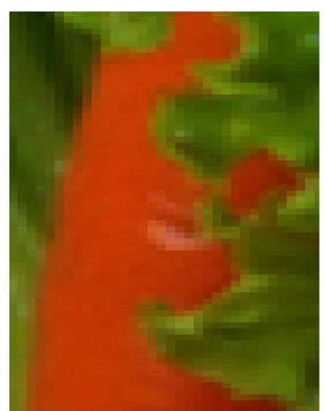

(f)

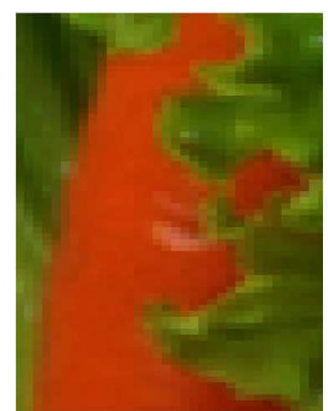

(g)

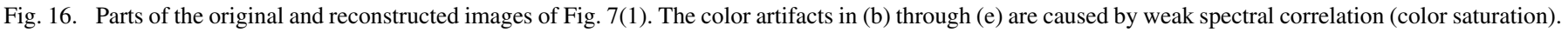

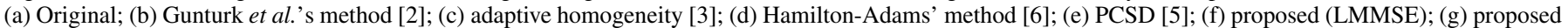
(SVR).

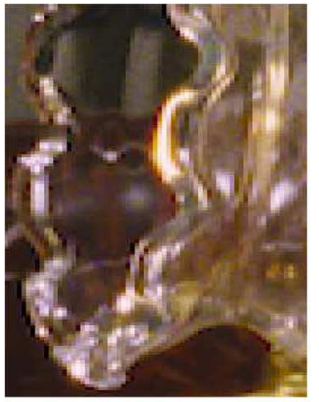

(a)

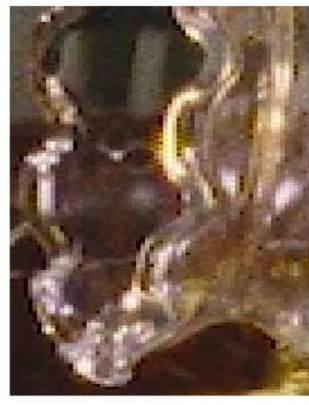

(b)

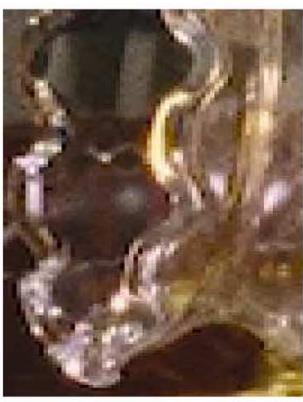

(c)

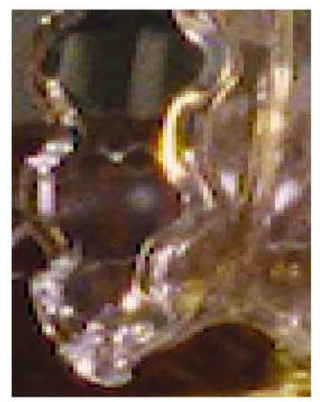

(d)

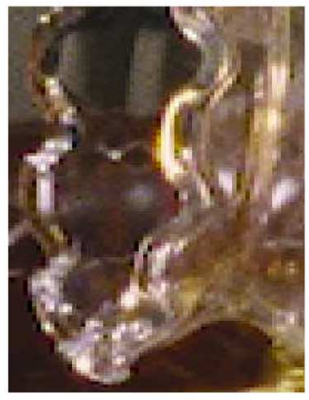

(e)

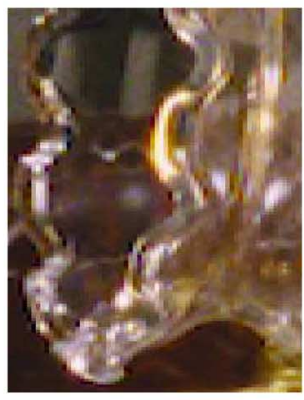

(f)

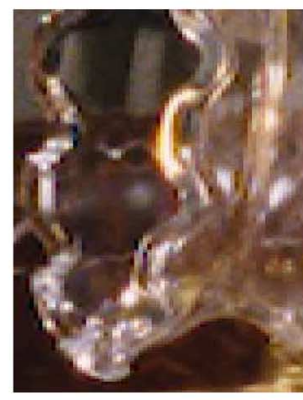

(g)

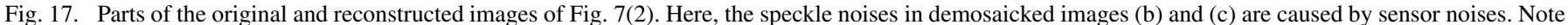

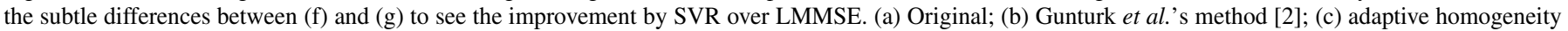
[3]; (d) Hamilton-Adams' method [6]; (e) PCSD [5]; (f) proposed (LMMSE); (g) proposed (SVR).

a better performance. This indicates the important role played by intraband interpolation in the proposed hybrid demosaicking framework.

\section{CONCLUSION}

We proposed a new hybrid approach of color demosaicking that combines inter and intraband estimates of missing sam- ples. The new approach cures a common flaw of existing demosaicking techniques: susceptibility to color artifacts in areas of weak spectral correlation or sensor noises. Two embodiments of the new approach, an LMMSE-based demosaicking technique and a SVR-based technique were developed and evaluated. Both of them eliminate visually objectionable color artifacts of existing color demosaicking methods, and achieve significantly higher PSNR and superior image quality. 


\section{ACKNOWLEDGMENT}

The authors would like to thank Prof. Y.-P. Tan and his group for providing their demosaicking software to conduct the comparison study.

\section{REFERENCES}

[1] B. E. Bayer, Eastman Kodak Company, "Color Imaging Array," U.S. Patent 3971 065, Jul. 20, 1976.

[2] B. K. Gunturk, Y. Altunbasak, and R. M. Mersereau, "Color plane interpolation using alternating projections," IEEE Trans. Image Process., vol. 11, no. 9, pp. 997-1013, Sep. 2002.

[3] K. Hirakawa and T. W. Parks, "Adaptive homogeneity-directed demosaicing algorithm," IEEE Trans. Image Process., vol. 14, no. 3, pp. 360-369, Mar. 2005.

[4] X. Li, B. Gunturk, and L. Zhang, "Image demosaicing: A systematic survey," SPIE, vol. 6822, pp. 68 221J-68 221J-15, 2008

[5] X. Wu and N. Zhang, "Primary-consistent soft-decision color demosaicking for digital cameras," IEEE Trans. Image Process., vol. 13, no. 9, pp. 1263-1274, Sep. 2004.

[6] J. F. Hamilton and J. E. A. , "Adaptive Color Plane Interpolation in Single Sensor Color Electronic Camera,” U.S. Patent 5629 734, May 13, 1997.

[7] X. Wu and L. Zhang, "Improvement of color video demosaicking in temporal domain," IEEE Trans. Image Process., vol. 15, no. 10, pp. 3138-3151, Oct. 2006.

[8] L. Chang and Y.-P. Tan, "Effective use of spatial and spectral correlations for color filter array demosaicking," IEEE Trans. Consum. Electron., vol. 50, no. 1, pp. 355-365, Jan. 2004.

[9] L. Chang and Y.-P. Tan, "Hybrid color filter array demosaicking for effective artifact suppression," J. Electron. Imag., vol. 15, 2006.

[10] X. Zhang and X. Wu, "Structure preserving image interpolation via adaptive $2 \mathrm{~d}$ autoregressive modeling," presented at the IEEE Int. Conf. Image Process., San Antonio, TX, 2007.

[11] V. Vapnik, Statistical Learning Theory. New York: Wiley, 1998.

[12] C. Kwan and X. Wu, "A classification approach to color demosaicking," in Proc. IEEE Int. Conf. Image Process., San Antonio, TX, Oct. 2004, vol. 4, pp. 2415-2418.

[13] C.-C. Chang and C.-J. Lin, LIBSVM: A Library for Support Vector Machines 2001 [Online]. Available: http://www.csie.ntu.edu.tw/cjlin/ libsvm

[14] X. Li and T. Orchard, "New edge-directed interpolation," IEEE Trans. Image Process., vol. 10, no. 10, pp. 1521-1527, Oct. 2001.

[15] RAW Photos From Digital Cameras (DSLRs) [Online]. Available: http://raw.fotosite.pl/

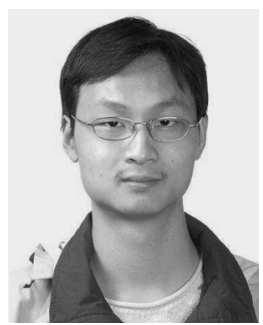

Fan Zhang received the B.E. degree in electrical engineering from the Shanghai Jiao Tong University, Shanghai, China, in 2007. He is currently pursuing the M. S. degree at Cornell University, Ithaca, NY.

$\mathrm{He}$ is currently pursuing the M.S. degree at the Institute of Image Communication and Information Processing, Department of Electronic Engineering, Shanghai Jiao Tong University. He visited McMaster University, Canada, in 2008 as a visiting student. His research interests are image and video processing problems, including demosaicking and superresolu-

tion, etc.

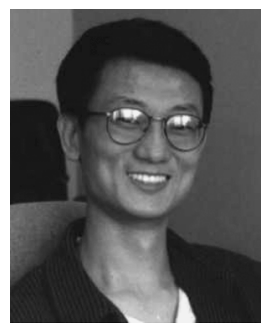

Xiaolin Wu (SM'96) received the B.Sc. degree from Wuhan University, Wuhan, China, in 1982, and the $\mathrm{Ph} . \mathrm{D}$. degree from the University of Calgary, Calgary, AB, Canada, in 1988.

$\mathrm{He}$ is currently a Professor with the Department of Electrical and Computer Engineering, McMaster University, Hamilton, ON, Canada, where he holds the NSERC-DALSA Research Chair in digital cinema. His research interests include multimedia coding and communications, image processing, signal quantization and compression, and joint source-channel coding. He has published over 160 research papers and holds two patents in these fields.

Dr. Wu received the 2003 Nokia Visiting Fellowship, the 2000 Monsteds Fellowship, and the 1998 UWO Distinguished Research Professorship. He is an associate editor of IEEE TRANSACTIONS ON MULTIMEDIA.

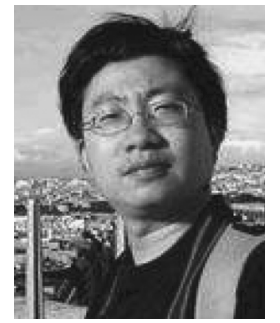

Xiaokang Yang (SM'00-SM'04) received the B.S. degree from Xiamen University, Xiamen, China, in 1994, the M.S. degree from the Chinese Academy of Sciences, Shanghai, China, in 1997, and the Ph.D. degree from Shanghai Jiao Tong University, Shanghai, in 2000 .

He is currently a Professor and the deputy Director of the Institute of Image Communication and Information Processing, Department of Electronic Engineering, Shanghai Jiao Tong University. From August 2007 to July 2008, he visited the Institute for Computer Science, University of Freiburg, Germany, as an Alexander von Humboldt Research Fellow. From September 2000 to March 2002, he worked as a Research Fellow at the Centre for Signal Processing, Nanyang Technological University, Singapore. From April 2002 to October 2004, he was a Research Scientist at the Institute for Infocomm Research (I2R), Singapore. He has published over 130 refereed papers, and has filed 14 patents. His current research interests include visual processing and communication, media analysis and retrieval, and pattern recognition. He actively participates in the International Standards of MPEG and JVT.

Dr. Yang received the Microsoft Young Professorship Award 2006, the Best Young Investigator Paper Award at IS\&T/SPIE International Conference on Video Communication and Image Processing (VCIP2003), and awards from the A-STAR and Tan Kah Kee foundations. He is currently a member of Design and Implementation of Signal Processing Systems (DISPS) Technical Committee of the IEEE Signal Processing Society and a member of Visual Signal Processing and Communications (VSPC) Technical Committee of the IEEE Circuits and Systems Society. He was the special session chair of Perceptual Visual Processing of IEEE ICME2006. He was the local co-chair of ChinaCom2007 and the technical program co-chair of IEEE SiPS2007.

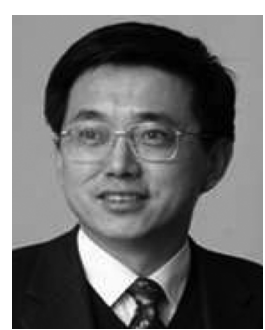

Wenjun Zhang (M'02) was born in Qingdao, China, in 1963. He received the B.S., M.S., and Ph.D. degrees in electronic engineering from Shanghai Jiao Tong University, Shanghai, China, in 1984, 1987, and 1989 , respectively.

From 1990 to 1993, He was a postdoctoral fellow at Philips Kommunikation Industrie AG in Nuremberg, Germany, where he was actively involved in developing HD-MAC (former European HDTV) system. He joined the Faculty of Shanghai Jiao Tong University in 1993 and became a full Professor in the Department of Electronic Engineering in 1995. As a team leader, he was successfully in charge of developing the first Chinese HDTV prototype system in 1998. By solving the technical bottleneck of high-speed mobile reception of digital television signals, he was one of the main contributors to the Chinese Digital Television Terrestrial Broadcasting Standard issued in 2006. He has had more than 37 patents granted and more than 80 referred papers published in international journals and conferences. His current research interests include digital video coding and transmission, multimedia semantic processing, and intelligent video surveillance.

Dr. Zhang is a Changjiang Scholarship Professor and was awarded by National Science Fund for Distinguished Scholars in the field of communications and information systems.

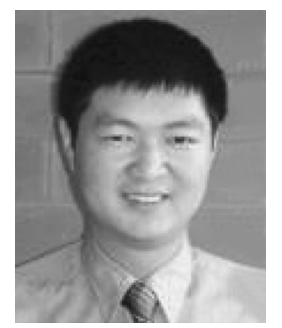

Lei Zhang (M'04) received the B.S. degree in 1995 from the Shenyang Institute of Aeronautical Engineering, Shenyang, China, and the M.S. and Ph.D degrees in Electrical and Engineering from Northwestern Polytechnical University, Xi'an, China, respectively, in 1998 and 2001.

From 2001 to 2002, he was a research associate with the Department of Computing, The Hong Kong Polytechnic University. From January 2003 to January 2006, he was a Postdoctoral Fellow in the Department of Electrical and Computer Engineering, McMaster University, Canada. Since January 2006, he has been an Assistan Professor in the Department of Computing, The Hong Kong Polytechnic University. His research interests include image and video processing, biometrics, pattern recognition, multisensor data fusion and optimal estimation theory, etc. 